Original Research Paper

\title{
Performance of Hybrid GANN in Comparison with Other Standalone Models on Dengue Outbreak Prediction
}

\author{
Nor Azura Husin, Norwati Mustapha, Md. Nasir Sulaiman, \\ Razali Yaacob, Hazlina Hamdan and Masnida Hussin \\ Department of Computer Science, Faculty of Computer Science and Information Technology, \\ University Putra Malaysia, Selangor, Malaysia
}

Article history

Received: 24-06-2015

Revised: 23-06-2016

Accepted: 01-07-2016

Corresponding Author:

Nor Azura Husin

Department of Computer

Science, Faculty of Computer

Science and Information

Technology, University Putra

Malaysia, Selangor, Malaysia

Email: support@thescipub.com

\begin{abstract}
Early prediction of diseases especially dengue fever in the case of Malaysia, is very crucial to enable health authorities to develop response strategies and context preventive intervention programs such as awareness campaigns for the high risk population before an outbreak occurs. Some of the deficiencies in dengue epidemiology are insufficient awareness on the parameter as well as the combination among them. Most of the studies on dengue prediction use standalone models which face problem of finding the appropriate parameter since they need to apply try and error approach. The aim of this paper is to conduct experiments for determining the best network structure that has effective variable and fitting parameters in predicting the spread of the dengue outbreak. Four model structures were designed in order to attain optimum prediction performance. The best model structure was selected as predicting model to solve the time series prediction of dengue. The result showed that neighboring location of dengue cases was very effective in predicting the dengue outbreak and it is proven that the hybrid Genetic Algorithm and Neural Network (GANN) model significantly outperforms standalone models namely regression and Neural Network (NN).
\end{abstract}

Keywords: Hybrid GANN, Genetic, Neural Network, Predicting

\section{Introduction}

Dengue is a tropical mosquito-borne disease affecting more than 100 countries worldwide. Current estimate by WHO put the number of cases at 50-100 million cases per-year while the most recent estimate by a multinational study just published in the Lancet, tripled the WHO estimate at 360 million cases per year with $40 \%$ of the world population at risk $(\mathrm{MOH}$, 2013). This disease may become the most important global health problem in the next decade which can no longer be ignored. This is further aggravated by environmental parameters like global warming, rapid urbanization and international traveling, which are recognized as contributing parameters to the spread of dengue outbreak (MOH, 2013). Dengue disease turns out to be the highest communicable disease compared to other prominent diseases like malaria, HFMD, typhus and yellow fever (MOH, 2012).

In Malaysia, dengue cases were categorized as a notifiable disease in 1971 . Since then, it continues to persist in predominantly urban and semi urban areas throughout the country. Approximately $70-80 \%$ of dengue cases are reported in areas where there is a high population density and rapid development activities which contribute to dengue transmission (Mahiran and Ho, 2011). Rapid urbanization has brought about enormous infrastructural build-up indirectly producing breeding areas for mosquito. Consequently, population growth and climate also considered as main parameters that contribute to the spike in dengue cases outbreak (Muhuiddin and Jamie, 2015).

Previous research already shows that the accuracy of prediction model can be better over standalone model if we combine several different models. The hybrid models are proved in order to search the suitable parameter and make it model more robust with regard to the possible structure change in the data. Although combining or hybrid model prove to be alternative on solving the previous problem, there is not many existing prediction model using hybrid model especially prediction on dengue outbreak. Therefore, this study propose that the 
hybrid model as the best model for predicting outbreak as it can solve previous problems in this research areas and provide better accuracy compared to those generated by other standalone model.

The aim of this paper is to conduct experiments for determining which model structure could deliver the best performance and thus, a more capable model for predicting the spread of dengue outbreak by comparing between a hybrid model of genetic algorithm and neural network and standalone models namely neural network and regression model. The rest of this paper is organized as follows; section 2 describes previous works. Designing model was explained in section 3. Section 4 discusses the implementation of hybrid GANN. Section 5 explained results and discussion of the case study. Section 6 describes our conclusion. Finally, some future works are summarized in Section 7.

\section{Previous Work}

Hybrid models are the combination of two or more model in one system that propose to solve the given problem. According to Gray and Kilgour (1997), hybrid model can be categorized by sequential, auxiliary and embedded hybrids which the sequential models make use of model in serial way. Meanwhile in auxiliary hybrid model, one model calls the other as a subroutine to process or manipulate information desired by it. Embedded hybrid models integrate the models in such a manner that they appear intertwined. The combination is so complete that in such manner that it would come out that no model can be used without the others for effective problem solving.

Table 1 summarizes the findings from previous researches on disease prediction models. Studies by Cen and Wang (2008) on predicting lung cancer using a hybrid Genetic Algorithm and Neural Network (GA-BP) demonstrated the hybrid model can speed up convergence to the optimal solution and provide an effectual model for early diagnosis of lung cancer. The result also shows that the hybrid of NN and GA can provide more accurate and efficient prediction compared to standalone model. Aburas et al. (2010) conclude that the combination of four parameters namely rainfall, number of dengue cases, mean temperature and relative humidity were very effective for predicting dengue cases. Besides, NN model have been found to be very effective processing systems for predicting dengue outbreak.

Another research by Rachata et al. (2008) also proposed NN and entropy model for predicting dengue hemorrhagic fever outbreak and the result demonstrated that it can achieve up to $85.92 \%$ of accuracy and concluded that the result is going better when using entropy transformation. Yusof (2011) develop a prediction model that incorporates LS-SVM and NN model in predicting dengue outbreak and the results demonstrated that LS-SVM produce the best result compare to $\mathrm{NN}$ in terms of predicting accuracy and computational time. Harrison and Kennedy (2005) developed and optimized NN model for diagnosis of acute coronary syndrome and concluded that $\mathrm{NN}$ is a suitable model for developing diagnosis algorithms for chest patients and this model calibrated and performed well on unseen data from different centers. Although some of them uses standalone model in their studies, they recommend applying a hybrid model to achieve the optimal parameter, thus improve the predicting accuracy.

These studies indicated that, hybrid model results outperform neural network, regression and even statistical methods using hybrid can provide better accuracy than standalone model because these methods prevent the NN from being stuck in the local minima (Shanti et al., 2009). Although the hybrid between GA and NN has delivered comparable results against other standalone approach however from our observation, there is rarely any study on the application of hybrid model for dengue outbreak predicting.

Table 1. The previous studies review on disease prediction model

\begin{tabular}{lll}
\hline Author & Research Domain & Method used \\
\hline Yusof (2011) & $\begin{array}{l}\text { Predict dengue outbreak based on dengue cases } \\
\text { data, rainfall data and proximity location data }\end{array}$ & $\begin{array}{l}\text { Least Square Support Vector Machines (LS-SVM) } \\
\text { and Neural Network Model }\end{array}$ \\
Aburas et al. (2010) & $\begin{array}{l}\text { Predict dengue cases on mean temperature, } \\
\text { Neural network }\end{array}$
\end{tabular}
mean relative humidity, total rainfall and total number of dengue cases

Cen and Wang (2008) Predict the lung cancer by introduces Multispecies Co-evolution Genetic Algorithm and

Hybrid Genetic Algorithm-BP Neural Networks Simulated Annealing algorithm.

Rachata et al. (2008) Propose an automatic prediction system of Dengue Hemorrhagic Fever (DHF) outbreak on weather condition and DHF cases data

Harrison and Kennedy To develop and optimize NN for diagnosis of (2005) acute coronary syndrome, to test the model on data collected prospectively from different centers.

Neural network and Entropy technique

Neural network and logistic regression. 


\section{Designing Model}

In the context of this study, the hybridization between neural network and genetic is attempted to overcome the weaknesses of one with the strengths of the other. Basically gradient descent learning is used in order to obtain their weights in standalone back propagation neural network. However there always remains the problem of the network becoming stuck in local minimum.

Therefore, this hybrid model which uses genetic algorithm to determine the weights is expected to achieve adequate result. Figure 1 illustrates the design of hybrid GANN model that applied in this study. The determinations of weight for hybrid GANN are performed by several procedures which consist of coding, weight extraction, fitness function and preproduction. The procedures of determinations of weight for hybrid GANN will be explained in next section.

\section{Implementation of Hybrid GANN Model}

To develop a hybrid model, the main procedure is to train the neural network to obtain the weights for a given set of input data and to infer output given a testing set using the weights obtained after training. This procedure involves two main files which are training file for train neural network and testing file for inferring the output of the given testing set respectively. This main procedure (training) will control all the procedures that must be executed in an exact order. All the main procedures will declare about seven sub procedures and these are: Weight extraction, produce genetic, convert weight, mating pool and fitness, cross sites and reproduce procedure.

The first procedure is to generate a population of random weights for the input-hidden and hidden-output layer of the models structure. At this stage we start with random number of generation. These models whose network configuration is $l-m-n$ where $l$ is input nodes, $m$ is hidden nodes and $n$ is output nodes. The numbers of weights to be determined are $(l+n) m$ with each weight were being a real number. We refer the number of gene length in the weight as $d$. Here $d=5$ is used for the number of digit allocated for each gene in the chromosome. The string $L=(l+n) m d$ represents the number of population size. An initial number of population sizes were generated randomly. The output of weight is made available at the specific file.

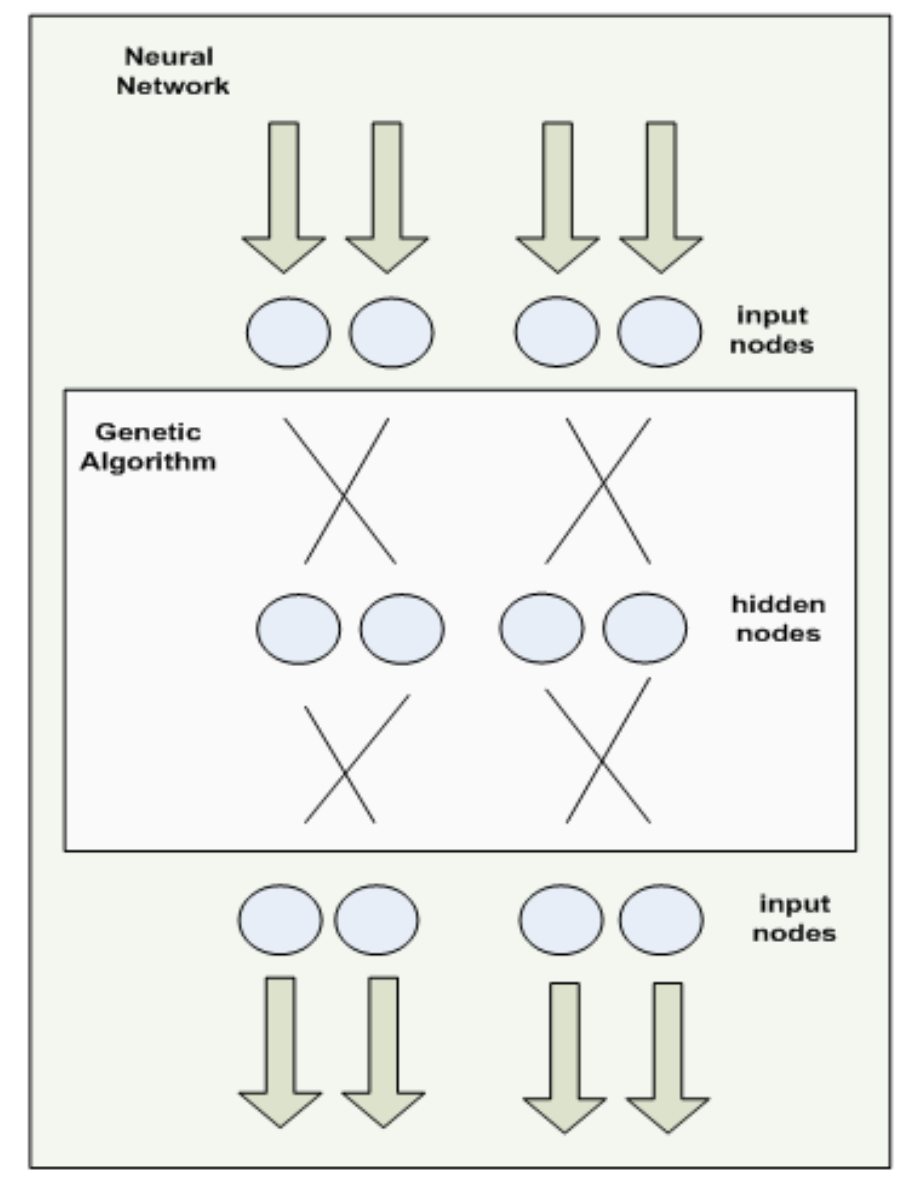

Fig. 1. The design of hybrid GANN model 
Table 2. The models structure with specific parameters

\begin{tabular}{lllll}
\hline Nodes & Struc 1 & Struc 2 & Struct 3 & Struc 4 \\
\hline Input $(l)$ & 4 & 8 & 8 & 12 \\
Output $(n)$ & 4 & 4 & 4 & 4 \\
Hidden nodes $(m)$ & 4,9 & 8,17 & 8,17 & 12,25 \\
Hidden layer & 1 & 1 & 1 & 1 \\
Weight $(w)$ & 16,32 & 48,96 & 48,96 & 96,192 \\
Population size $(L)$ & 80,160 & 240,480 & 240,480 & 480,960 \\
\hline
\end{tabular}

The number of input, hidden, output node and population size is determined based on configuration of the models structure as shown in Table 2.

For Structure 1, the input nodes is $l=4$ and output is $n=4$ nodes, hidden nodes are $m=4$ and 9 with one hidden layer. Meanwhile the weight $w$ are 16 and 32; and the population size are $L=80$ and 160 . Structure 2, the input is $l=8$ and output is $n=4$ nodes, hidden nodes are $m=8$ and 17 with one hidden layer. Meanwhile the weight $w$ are 48 and 96; and the population size is $L=240$ and 480 . For Structure 3, the input nodes is $l=8$ and output is $n=4$ nodes, hidden nodes are $m=8$ and 17 with one hidden layer. Meanwhile the weight $w$ are 48 and 96; and the population size are $L=240$ and 480 . Structure 4 , the input nodes is $l=12$ and output is $n=4$ nodes, hidden nodes are $m=12$ and 25 with one hidden layer. Meanwhile the weight $w$ are 96 and 192; and the population size are $L=480$ and 960 .

The next process is to produce the genetic that represents the population of chromosomes. This procedure will merge input-hidden weight and hiddenoutput weight files and compute as a genetic. Weight for each member of the population will emerge on a single line to favor the application of genetic operations.

To define the values of fitness for each chromosome then we need to extract weights from each of the chromosomes. The next procedure is to extract weight procedure that proposes to convert the weights to be in the range of -5 to +5 , existing in genetic file. This procedure will produce the final weight in other files. The number of population $m$, weight $w$ and gene length $d$ are determined based on configuration of the models structure as mention before. The actual weight $\mathrm{w}_{\mathrm{k}}$ is given by this Equation 1 (Rajasekaran and Vijayalakshmi Pai, 2008):

$$
w_{k}=\left\{\begin{array}{l}
+\frac{x_{k d+2} 10^{d-2}+x_{k d+3} 10^{d-3}+\ldots x_{(k+1) d}}{10^{d-2}}, \text { if } 5 \leq x_{k d+1} \leq 9 \\
-\frac{x_{k d+2} 10^{d-2}+x_{k d+3} 10^{d-3}+\ldots x_{(k+1) d}}{10^{d-2}}, \text { if } 0 \leq x_{k d+1}<5
\end{array}\right.
$$

Where:

$$
\begin{aligned}
x_{1}, x_{2}, \ldots, x_{d}, \ldots x_{L} & =\text { Represent a chromosome } \\
x_{k d+1}, x_{k d+2}, \ldots, x_{(k+1) d}= & \text { Represent the } k \text { th gene }(k \geq 0) \text { in } \\
& \text { the chromosome. }
\end{aligned}
$$

The fitness function must be formulated for each problem to be solved. In the first place, we need to check the fitness file to ensure convergence of fitness. If the convergence has not been attained, run the training procedure and call for the rebuild option. Rebuild begins generation from the last formed population of chromosomes and their acquired fitness. Meanwhile, if the convergence has been attained, testing procedure must be performed to infer the output of the testing data set.

Mating pool made by eliminating that chromosome $C_{l}$ with the least fitness $F_{\min }$ and change with duplicate copy of the chromosome $C_{k}$ that reporting the highest fitness $F^{\max }$. The parents are selected in pair at random. The offspring from the current population have their fitness calculated over again. In this study, the initial population of chromosome $P_{0}$ generated earlier with $F_{i}$, where $i=1,2 \ldots n$, as their fitness values.

\section{Results and Discussion}

\section{Result of Hybrid GANN Model}

Table 3 illustrated the results of error and recognition rate for model structure 1, 2, 3 and 4 by using Hybrid GANN Model. As we can see at this table model structure 3 that comprise between the cases of dengue data with dengue cases at nearby location significantly show the lowest error compared with others model structure produce in significant.

At Hulu Langat district, the best MSE error with is 0.077524 with recognition rate $92.25 \%$ with the combination of hidden nodes eight, hidden layer one, eight input and four output. At Hulu Selangor the combination of hidden nodes eight, hidden layer one, eight input and four outputs produce the best MSE error with 0.060782 with recognition rate $93.92 \%$. At Klang, the combination of hidden nodes 17 , hidden layer one, eight input and four output produce the best MSE error with 0.065243 with recognition rate $93.48 \%$.

Meanwhile, at Kuala Selangor the best MSE error is 0.065112 with recognition rate of $93.49 \%$ with the combination of hidden nodes eight, hidden layer one, eight input and four output. At Sepang, the combination of hidden nodes eight, hidden layer one, eight input and four output produce the best MSE error with 0.068891 with recognition rate $93.11 \%$. 


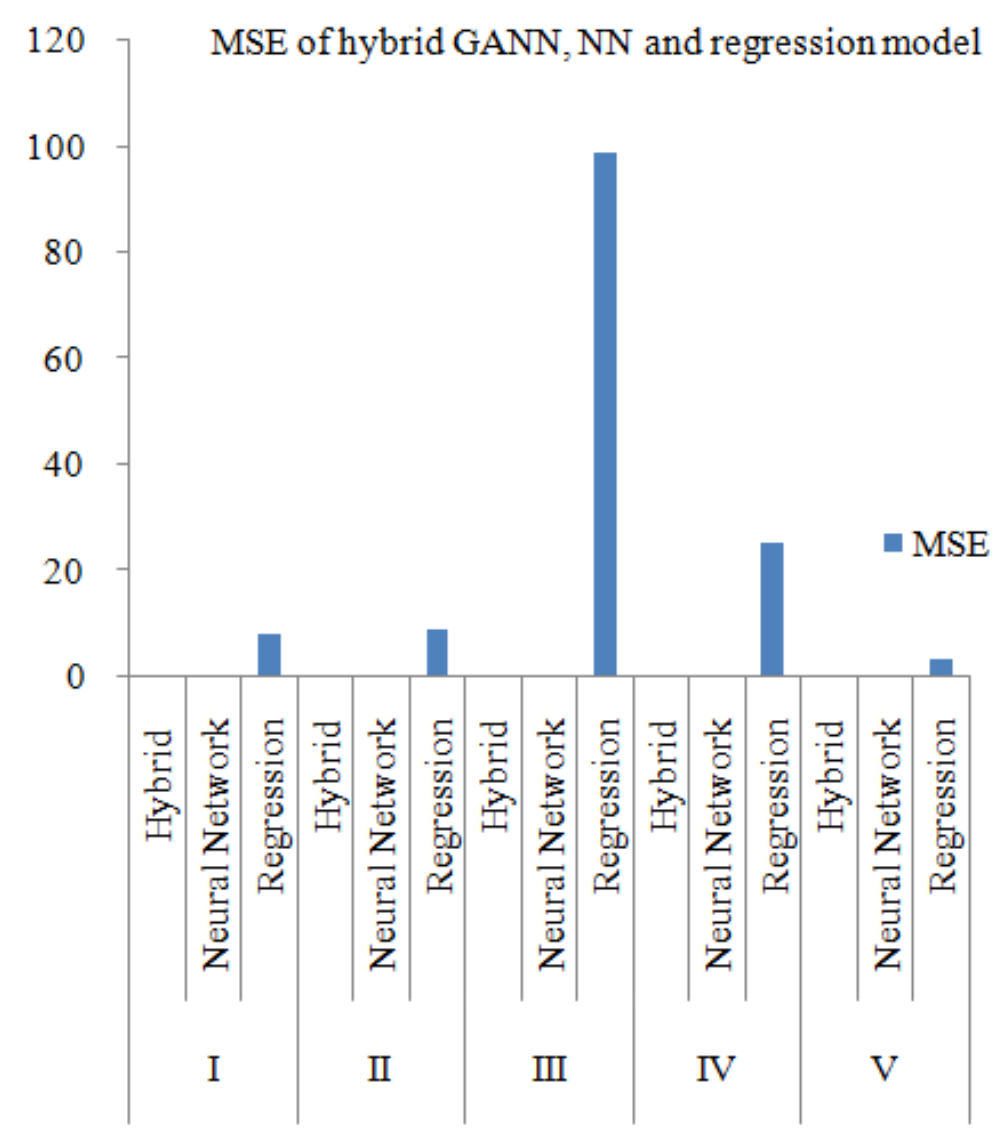

Fig. 2. Comparison result of MSE by using hybrid GANN, NN and regression model

Table 3. Results of recognition rate for model structure 1, 2, 3 Structure and

and 4

I

\begin{tabular}{|c|c|c|c|}
\hline Structure & District & Error & Rate \\
\hline \multirow[t]{5}{*}{1} & I & 0.111665 & 88.33 \\
\hline & II & 0.06268 & 93.73 \\
\hline & III & 0.06589 & 93.41 \\
\hline & IV & 0.085603 & 91.44 \\
\hline & $\mathrm{V}$ & 0.07681 & 92.32 \\
\hline \multirow[t]{5}{*}{2} & I & 0.072206 & 92.78 \\
\hline & II & 0.06498 & 93.5 \\
\hline & III & 0.07062 & 92.94 \\
\hline & IV & 0.070689 & 92.93 \\
\hline & V & 0.077099 & 92.29 \\
\hline \multirow[t]{5}{*}{3} & I & 0.068891 & 93.11 \\
\hline & II & 0.06078 & 93.92 \\
\hline & III & 0.07752 & 92.25 \\
\hline & IV & 0.065243 & 93.48 \\
\hline & $\mathrm{V}$ & 0.065112 & 93.49 \\
\hline \multirow[t]{5}{*}{4} & I & 0.078962 & 92.1 \\
\hline & II & 0.06511 & 93.49 \\
\hline & III & 0.07506 & 92.49 \\
\hline & IV & 0.066073 & 93.39 \\
\hline & V & 0.088945 & 91.11 \\
\hline
\end{tabular}

rict

Error

Table 4. Result of hybrid GANN, neural network and regression

\begin{tabular}{lll}
\hline District & Model & Error \\
\hline I & Hybrid & 0.068891 \\
& Neural Network & 0.073620 \\
II & Regression & 7.779000 \\
& Hybrid & 0.060780 \\
& Neural Network & 0.064100 \\
III & Regression & 8.667000 \\
& Hybrid & 0.077520 \\
& Neural Network & 0.081930 \\
IV & Regression & 98.54900 \\
& Hybrid & 0.065243 \\
& Neural Network & 0.073900 \\
V & Regression & 25.14700 \\
& Hybrid & 0.065112 \\
\multicolumn{1}{c}{ Neural Network } & 0.069770 \\
I = Sepang, II = Hulu Selangor, III = Hulu Langat, IV $=$ Klang \\
and V = Kuala Selangor \\
\multicolumn{2}{l}{ Regression Model }
\end{tabular}

The main objective of this research is to determine which model structure will produce the best prediction 
of the spread of dengue outbreak; which Structure 1 involved only the cases of dengue data, Structure 2 that comprise of dengue cases and rainfall data, Structure 3 that comprise of dengue cases and nearby location of dengue cases data and Structure 4 that include all the variables. Table 4 below illustrated the results between Hybrid GANN, NN and Regression Model in Kuala Selangor, Hulu Selangor, Sepang, Klang and Hulu Langat district based on the experimentation. From this table we can conclude that the Hybrid GANN model outperforms other standalone models. Figure 2 obviously shows that the regression model produce the highest error at all location.

\section{Conclusion}

This paper conduct experiments for finding which model structure will be the best for predicting the spread of dengue outbreak by comparing the performance of a hybrid model with conventional models namely neural network and regression model. The result from this experiment showed that the hybrid GANN model provides more accurate and efficient prediction compared to other standalone models. The result also showed that the model structure 3 that comprise with dengue cases and data on neighboring location was very effective in predicting dengue outbreak.

Moreover, this study has shown that the relative capability of hybrid model in dengue outbreak prediction and assisted to recognize the suitable variable and parameter for this experiment and their effect on predicting result. Hence, this study can be used as an indication for future research, especially in the context of prediction by comparing it with the performance of other models.

\section{Future Work}

Further research will be carried out to improve our dengue by observing other two parameters that persuaded the hybrid model performance and also explore different time frame. Besides, since the epidemic can be caused by different factors, we suggest exploring other factors that are not included in this research.

\section{Acknowledgement}

Much appreciation goes to the Department of Meteorological and Health State of Selangor, Malaysia and for providing information and relevant data for this study.

\section{Author's Contribution}

Nor Azura Husin: Collected, interpreted and analyzed the data and also implemented the model using $\mathrm{C}++$ programming language. Mainly wrote the first draft of the manuscript.

Norwati Mustapha: Designed the main concepts and planned the steps of the proposed model. Final approval of the revision to published.

Md. Nasir Sulaiman and Razali Yaacob: Designed the main concepts and planned the steps of the proposed model.

Hazlina Hamdan and Masnida Hussin: Reviewed and analytically evaluated the manuscript for submission.

\section{Ethics}

This article is original and contains unpublished material. The corresponding author approved the manuscript and confirms that no ethical issues involved.

\section{References}

Aburas, H.M., B.G. Cetiner and M. Sari, 2010. Dengue confirmed-cases prediction: A neural network model. J. Expert Syst. Applic., 37: 4256-4260.

DOI: $10.1016 /$ j.eswa.2009.11.077

Cen, L. and M. Wang, 2008. Application of hybrid genetic algorithm-BP neural networks to diagnosis of lung cancer. Proceedings of the International Conference on Computer Science and Software Engineering, Dec. 12-14, IEEE Xplore Press, Wuhan, Hubei, pp: 36-39.

DOI: $10.1109 /$ CSSE.2008.524

Gray, A. and R. Kilgour, 1997. Hybrid system FAQ. Version Draft 1.00.

Harrison, R.F. and R.L. Kennedy, 2005. Artificial neural network models for prediction of acute coronary syndromes using clinical data from the time of presentation. Ann. Emergency Med., 46: 143-439. DOI: 10.1016/j.annemergmed.2004.09.012

Mahiran, M. and B.K. Ho, 2011. Clinical practice guideline on management of dengue infection in adult. J. Malaysian Family Phys.

$\mathrm{MOH}, 2013$. Malaysia calls for more concerted global R\&D effort against dengue at 66th WHO Assembly, Switzerland. Ministry of Health Malaysia, Official Press Statement by the Deputy Director General.

$\mathrm{MOH}, 2012$. Health fact 2012, Health of Informatics Centre Planning and Development Division $\mathrm{MOH} / \mathrm{S} / \mathrm{RAN} / 31.12$ (TR).

Muhuiddin, H. and T. Jamie, 2015. Variables that may affect the transmission of dengue-a case study for health management in Asia. Topics in Public Health. 
Rachata, N., P. Charoenkwan, T. Yooyativong, K. Chamnongthai and C. Lursinsap et al., 2008. Automatic prediction system of dengue haemorrhagic-fever outbreak risk by using entropy and artificial neural network. proceedings of the International Symposium on Communications and Information Technologies, Oct. 21-23, IEEE Xplore Press, Lao, pp: 210-214.

DOI: 10.1109/ISCIT.2008.4700184

Rajasekaran, S. and G.A. Vijayalakshmi Pai, 2008. Neural networks, fuzzy logic and genetic algorithms: Synthesis and applications. PHI Learning Private Limited.
Shanti, D., G. Sahoo and N. Saravanan, 2009. Evolving connection weights of artificial neural networks using genetic algorithm with application to the prediction of stroke disease. Int. J. Soft Comput., 4: 95-102.

Yusof, H., 2011. Dengue outbreak prediction: A least squares support vector machines approach. Int. J. Comput. Theory Eng., 3: 489-493.

DOI: 10.7763/IJCTE.2011.V3.355 